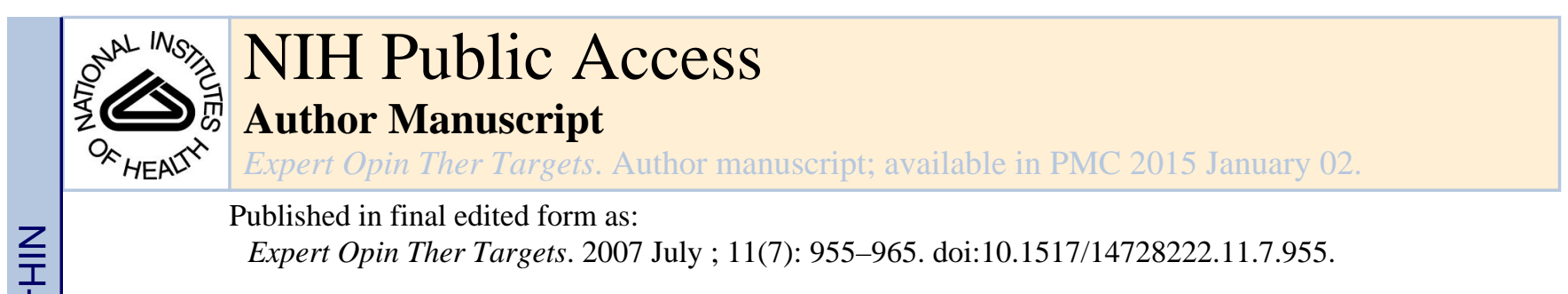

\title{
FOXP1: a potential therapeutic target in cancer
}

\author{
Henry B Koon ${ }^{1}$, Gregory C Ippolito ${ }^{2}$, Alison H Banham ${ }^{3}$, and Philip W Tucker ${ }^{\dagger, 2}$ \\ ${ }^{1}$ Division of Hematology/Oncology, Beth Israel Deaconess Medical Center, Harvard Medical \\ School, MA, USA \\ ${ }^{2}$ University of Texas, Department of Molecular Genetics and Microbiology, Institute of Cellular \\ and Molecular Biology, 1 University Station A5000, Austin, Texas 78712-0162, USA \\ ${ }^{3}$ University of Oxford, Nuffield Department of Clinical Laboratory Sciences, John Radcliffe \\ Hospital, Headington, Oxfordshire, OX3 9DU, UK
}

\section{Abstract}

Forkhead Box P1 (FOXP1) is a member of the FOX family of transcription factors which have a broad range of functions. Foxp1 is widely expressed and has been shown to have a role in cardiac, lung and lymphocyte development. FOXP1 is targeted by recurrent chromosome translocations and its overexpression confers a poor prognosis in a number of types of lymphomas, suggesting it may function as an oncogene. In contrast, FOXP1 localises to a tumour suppressor locus at 3p14.1 and loss of FOXP1 expression in breast cancer is associated with a worse outcome, suggesting FOXP1 may function as a tumour suppressor in other tissue types. These data suggest that FOXP1 may not only be useful in prognosis but also may be used to develop FOXP1-directed therapeutic strategies.

\section{Keywords}

3p; forkhead; Foxp1; lymphoma; oncogene; transcription factor; tumour suppressor

\section{Introduction}

Transcription factors play a critical role in the pathogenesis of cancer. The balanced translocation in Burkitt's lymphoma that results in dysregulated expression of $M y c$ is the classic example of a transcription factor functioning as an oncogene [1]. Although $C / E B P a$ plays a critical role in myeloid cell development, its loss or inactivation in non-small cell lung cancer and leukaemia suggests it functions as a tumour suppressor in many tissue types [2]. Similarly, the Forkhead Box P1 (Foxpl) transcription factor, may function as an oncogene or as a tumour suppressor depending on the cell type [2-5].

Foxpl is a member of the broadly expressed Foxp subset of "forkhead" (Fox) transcription factors $[6,7]$. Foxp proteins play critical roles in immune responses, organ development and cancer pathogenesis [6,7]. As with other members of this family, Foxp1 has a diverse

\footnotetext{
(C) 2007 Informa UK Ltd

${ }^{\dagger}$ Author for correspondence Tel: +1 512475 7705; Fax: +1 512475 7707; philtucker@mail.utexas.edu.
} 
repertoire of functions ranging from the regulation of B-cell development and monocyte differentiation to the facilitation of cardiac valve development and lung development [8-13]. Thus, it is not surprising that FOXP1 also appears to play a role in malignancy $[3-5,14,15]$. Foxp1 was initially discovered in a screen to detect glutamine-rich transcription factors in B cells and named glutamine (Q)-rich factor $1(\mathrm{QRF} 1)$ [16]. Later, a group cloned the human FOXP1 gene by screening cDNA expression libraries to determine the specificity of the JC12 antibody, which recognised a previously unknown protein that displayed a predominantly nuclear subcellular localisation and that was widely expressed in normal human tissues [3].

FOXP1 transcriptional activities appear to be inhibited in a large number of epithelial malignancies by either a decrease in FOXP1 mRNA, a decrease in FOXP1 protein levels, or by aberrant localisation of FOXP1 to the cytoplasm [3]. Location of FOXP1 on chromosome 3 p14.1, a region that is associated with a loss of heterozygosity in a number of tumour types, has further implicated FOXPI as a potential tumour suppressor gene [17,18]. Further investigation of the role of FOXP1 in haematological malignancies has revealed a more complicated picture. FOXP1 has been found to be overexpressed in a variety of B-cell lymphomas $[4,5,19]$. In some cases this overexpression was the result of a chromosomal translocation that resulted in the unregulated expression of FOXP1 [12,13]. In several B-cell non-Hodgkin lymphomas, as opposed to epithelial malignancies, FOXP1 appears to have all of the hallmarks of an oncogene. In this review we will focus on what is known about the biology of Foxp1 in the context of the Foxp subfamily, the role of FOXP1 in the development of cancer, and potential FOXP1-based strategies for cancer therapeutics.

\subsection{Diverse functions of Foxp transcription factors}

There are 43 FOX genes in the human genome. Many, including the four Foxp sub-family genes, are essential for development and organogenesis [6]. For example, FOXP2 was the first genetic locus discovered to be impaired in inherited defects of speech and language development [20,21]. Point mutation of a single amino acid within the forkhead domain of FOXP2 was observed in afflicted individuals, and this was proposed to be the cause of their severe speech defects [21]. Foxp2 null mice die postnatally as a result of defective lung alveolarisation [9]. FOXP3 was discovered as the gene mutated in X-linked autoimmunityallergic dysregulation syndrome and plays a pivotal role in the immune system in maintenance of self-tolerance [22-24]. Loss of FOXP4 expression was observed in human larynx carcinomas and kidney tumours [25]. Foxp4 null embryos exhibit a delay in anterior foregut closure, a defect resulting in cardia bifida [26].

Foxp1 has a critical and unique role in cardiac valve morphogenesis of mice [10]. Expression of Foxp1 in the myocardium and endocardium is observed from E9.5 to E14.5 [10]. Foxp1-positive cells underlying the cushion mesenchyme are observed at E11.5 but disappear from this region later in development. Foxp1 expression is retained in the overlying endocardium throughout development [10]. Elimination of Foxpl by targeted deletion in mice results in embryonic death at E14.5 with severe defects in ventricular and outflow tract septation, endocardial cushion development, and cardiac myocyte proliferation and maturation [10]. This phenotype is strikingly similar (e.g., stage of embryonic lethality, 
defective cardiac septation and endocardial cushion deformation) to that observed for NFAT2/1c knockout mice. This and other data suggest a potential interaction between these two transcriptional pathways $[10,27]$.

\subsection{Structure and function of Foxp subfamily members}

All Fox transcription factors bind to DNA through a forkhead or "winged helix" domain (Figure 1) located near their carboxyl-termini [16]. Foxp1 forkheads contain deletions (compared with other Fox forkheads) in their "wing" regions [16]. Also in contrast with other forkhead subfamilies, Foxp members share domains and homologies that extend beyond the forkhead DNA-binding domain $[9,28]$. These include a $\mathrm{C} 2 \mathrm{H} 2$ zinc finger and leucine zipper motif that promotes self- and inter-parlogous dimerisation. Other Fox proteins typically exist as monomers in solution and when bound to DNA [16]. The ability to undergo heteromerisation extends the range of biological functions of the Foxp subfamily $[9,28]$.

Foxp proteins generally function as transcriptional repressors [9,28,29]. For example, Foxp1 and Foxp3 repress $\mathrm{Ca}^{2+}$-stimulated activity of the IL-2 promoter [9,30-32]. With the exception of Foxp3, Foxp proteins contain polyglutamine tracts near their $N$-terminal [9,28]. Alternative inclusion or exclusion of the polyQ track via pre-mRNA splicing alters the ability of Foxp1 to repress IL-2 [28]. Foxp 1 interacts with $C$-terminal binding protein 1 $(C \mathrm{tBP} 1)$ to enhance its transcriptional repression on model promoters and with the multifunctional hnRNP protein, nonO/p54nrb (non-POU domain containing, octamer-binding/ nuclear RNA-binding protein, $54 \mathrm{kDa}$ ), a marker of nuclear paraspeckles [28,29,33].

FOXP family proteins, including FOXP1, also form cooperative complexes with NFAT2 when bound to the same region of the IL-2 promoter [34]. The crystal structure of the FOXP2 forkhead domain-NFAT2 DNA complex revealed an extensive interface. All NFAT and DNA contacts of FOXP2 are conserved with FOXP1 and FOXP3 (Figure 1) [34]. NFAT function is often determined by its interaction with other transcription factors. Most prominently, NFAT:AP-1 (Fos/Jun) interaction on cooperative DNA binding sites, including the IL-2 promoter, controls apoptosis, proliferation and cytokine expression $[35,36]$.

\subsection{Foxp1 in B-cell development}

The critical role of NFAT normal lymphocyte biology and the interaction between NFAT and FOXP1 suggested that FOXP1 may also have a role in lymphocyte development [34]. Indeed, Foxp1 transcripts are most highly expressed in hematopoietic cells [101]. Within the B lineage, Foxp1 is expressed modestly in progenitors, with highest levels in activated B cells and mantle zone B cells $[4,12,13,37]$.

To circumvent embryonic lethality and to determine its role in B cell development/function, $R A G$-deficient mice were reconstituted with Foxp $^{-/-}$E14.5 fetal liver cells [8]. While Tcell development was completely normal, B cells in the lymph nodes and spleens of Foxp $1^{-1-}$ recipients were dramatically reduced [8]. Flow cytometry analysis of bone marrow indicated blockade at the pro-B cell stage. Following culture of ${\text { Foxp } 1^{-/}}^{-}$transplanted bone marrow with S17 stromal cells and IL-7, no cells advanced beyond B220 $0^{+} \mathrm{CD} 43^{+}$(Fraction 
B) and their proliferation was significantly reduced. These data suggested an intrinsic proliferation defect in $\mathrm{Foxpl}^{-/-}$pro-B cells, as the IL-7R expression levels were normal. Significant reduction of intracellular $\mu+$ cells indicated a potential defect in variable, diversity, joining (VDJ) recombination. $\mathrm{DJ}_{\mathrm{H}}$ joint formation and germline transcription of both distal $\left(\mathrm{V}_{\mathrm{H}} \mathrm{J} 558\right)$ and proximal $\left(\mathrm{V}_{\mathrm{H}} 7183\right) \mathrm{V}_{\mathrm{H}}$ families were severely diminished in Foxp $^{-/-}$pro-B cells [8]. Expression levels of both RAG1 and RAG2 were reduced to $~ 10 \%$ of control. Transcript levels of key regulators/components [38,39] of B-cell development and VDJ rearrangement (e.g., E2A, EBF, Pax5 Iga, $\lambda 5$, VpreB) were markedly reduced in pro-B cells isolated directly from fetal liver or when these cells were cultured in vitro. There are four consensus Foxp1 binding sites in the $5^{\prime}$ end of the common RAG intergenic enhancer $\left(\mathrm{E}_{\mathrm{RAG}}\right)$. Chromatin immunoprecipitation and electrophoretic mobility shift assay assays demonstrated that Foxp1 is recruited to at least two of these sites in normal B220 $\mathrm{B}$ cells but not in thymocytes [8].

It is important to note that the Foxp1-mediated pro-B block was not complete, as a small number of $\operatorname{IgM}^{+} \operatorname{IgD}^{+}$Foxp $^{-/-}$B cells were observed in the spleen [8]. This suggested a role for Foxp1 at a more mature stage of B-cell development, such as that targeted in the transformed state of lymphoma. Perhaps the timing and cell-type specificity of Foxp1 function depends, at least in part, on its association with factors such as NFAT.

\subsection{Foxp transcription factors in T-cell development and function}

Regulatory $\mathrm{T}$ cells $\left(\mathrm{T}_{\text {regs }}\right)$, which play a pivotal role in the maintenance of self-tolerance, were originally characterised as CD4 ${ }^{+} \mathrm{T}$ cells constitutively expressing CD25 [40,41]. Foxp3 is specifically expressed in $\mathrm{T}_{\text {regs }}$ and functions as a "master regulator" of their lineage development and function [42-45]. Foxp3-mediated suppression was shown to be mediated at the transcriptional level by NFAT-AP-1 following ligation of the T-cell receptor [30,31]. The significance of this was not appreciated until recently. An elegant series of experiments by Wu et al. demonstrated that mutation of FOXP3 forkhead residues which disrupted NFAT-FOXP3 interaction (Figure 1) impaired the ability of FOXP3 to repress $I L-2$ and the other prototypic $\mathrm{T}_{\text {reg }}$ markers, such as CTLA4 [34]. They further showed that retrovirally transduced $\mathrm{T}_{\text {regs }}$ that over-expressed these mutated Foxp3 proteins failed to suppress function in a mouse model of autoimmunity [34].

Although thymic development in $F_{\text {oxp }}{ }^{-/-}$-reconstituted $R A G^{-/-}$mice is normal [8], a subsequent Foxp1 function in effector T-cell activation and/or subset differentiation has not been addressed in vivo. The physical interaction of Foxp1 with Foxp3 and their association in NFAT-IL-2 promoter DNA complexes [28,34] indicate that this will be an informative avenue to pursue. The vital importance of in vivo cooperativity among Foxp transcription factors is further indicated by recent data demonstrating that Foxp2 or Foxp3 deficiency can lead to compensatory function or altered expression, respectively, of Foxp1 [46,47].

\section{The role of FOXP1 in cancer}

\subsection{FOXP1: tumour suppressor}

Loss of heterozygosity of chromosome $3 p$ is a common genetic event in multiple cancers that has led to the discovery of a number of tumour suppressor genes in this region $[17,18]$. 
The location of FOXP1 on 3p14.1 had led to its proposed candidacy as a tumour suppressor [3]. Among the other candidate tumour suppressor genes, DUTT1/ROBOI (deleted-in-Utwenty-twenty) (3p12-13), FHIT (fragile histidine triad) (3p14.1), Ras-association domain family 1 (3p21.1) and VHL (Von Hippel Lindau, 3p25) have been shown to be deleted, inactivated by methylation or functionally altered by point mutations in human cancers $[17,18]$. Additionally, mice with targeted deletions of FHIT and DUTTI/ROBOI show an increased frequency of spontaneous and chemically induced tumours compared with control mice, supporting their status as tumour suppressor genes [48,49]. FOXP1 is differentially expressed in a number of malignant cell types and is thus another potential tumour suppressor gene on $3 \mathrm{p}$.

2.1.1 Gastrointestinal cancers-In the initial studies of FOXP1, RNA expression data showed that FOXP1 was expressed in virtually every tissue examined [3]. Interestingly, RNA expression levels in over half of tumour samples examined showed differential expression [3]. This finding was most prominent in colon cancer specimens where 6 out of 11 samples demonstrated decreased expression compared with controls in a tumour sample array [3]. Decreased FOXP1 protein expression was seen in malignant cells compared to normal colonic tissue in 18 out of 20 cases by immunohistochemistry [3]. In this series, 15 out of the 18 samples had weak or absent nuclear staining with the FOXP1 antibody [3]. In contrast, stomach cancer samples on a tumour expression array had elevated levels of FOXP1 mRNA in 6 out of 8 cases [3]. However, when protein levels were examined by immunohistochemistry in a separate series of 21 patients, 14 specimens showed either absent or weak nuclear and/or cytoplasmic staining, and the remaining 7 cases had strong nuclear staining [3]. Only five cases of pancreatic cancer were evaluated in this initial report, and each had weak nuclear staining compared with normal tissue [3]. Three of these cases stained strongly with the FOXP1 antibody but the expression was primarily cytoplasmic, suggesting that aberrant localisation may be a mechanism for inactivation of FOXP1 [3]. No followup studies have been published, to date, on these findings in colon cancer or the other gastrointestinal malignancies, and the impact of FOXP1 on survival and response to treatment in these diseases remains to be determined.

2.1.2 Lung, head and neck cancers-A potential role for FOXP1 in lung cancer is tantalising, due to its expression pattern during lung development [3] and due to the fact that genetic aberrations of $3 \mathrm{p}$ are reportedly seen in $>90 \%$ of non-small cell lung cancer (NSCLC) and almost 100\% of small-cell lung cancer (SCLC) [18]. In the initial report that suggested FOXP1 was a tumour suppressor, 1 out of 3 lung cancers from an expression array showed decreased expression [3]. At the protein level, none of the 6 cases of NSCLC examined showed nuclear staining, and only 2 out of 6 had weak cytoplasmic staining. In the 6 cases of SCLC examined, 2 had weak nuclear staining, and the remainder were patchy or negative [3]. In head and neck cancer, moderate FOXP1 nuclear levels were seen in 2 out of 10 samples examined. Nuclei of five of the remaining samples stained weakly, whereas the remainder had no nuclear FOXP1 staining [3]. Interestingly, in a nitrosamine-induced rat lung cancer model, Foxpl and Rassfia, another putative tumour suppressor gene, were lost in the cancers that developed [50]. This group had already identified alterations in Fhit and 
Dutt1/Robol in this model, highlighting the need for more evidence before one can conclude that Foxpl is a tumour suppressor and not just a marker for $3 p$ loss.

2.1.3 Genitourinary malignancies-The expression of FOXP1 has been examined in renal cell carcinoma, prostate cancer and endometrial cancer. In renal cell carcinoma, all cases examined had FOXP1 protein expression [3]. Nuclear staining with FOXP1 was seen in 7 out of 10 cases, including 3 out of 4 cases with VHL mutations, another 3p tumour suppressor gene [3]. Prostate cancer revealed a more complex picture. Prostate intraepithelial neoplasia (PIN), a preinvasive lesion, showed increased FOXP1expression compared with normal tissue as judged by immunohistochemistry [3]. However, immunochemical analyses demonstrated that 5 out of 35 cases of prostate cancer showed increased Foxp1 expression, whereas 9 of 35 cases had no expression of FOXP1 [3]. A follow-up study demonstrated that nuclear, but not cytoplasmic, expression of FOXP1 correlated with expression of androgen receptors, HIF1-a, HIF2- $\alpha$ and vascular endothelial growth factor [51]. The expression of FOXP1 did not appear to be regulated by androgens or hypoxia in prostate cell lines [51]. Unfortunately, there is no data yet on the impact of FOXP1 expression on survival or the clinical effectiveness of androgen antagonists in prostate cancer.

Interestingly, exclusively cytoplasmic localisation of FOXP1 in endometrial cancer, as assessed by immunohistochemistry, correlated with increased HIF1-a expression and deep myometrial invasion [15]. This study did not investigate whether any of the other tumour suppressor genes on chromosome $3 p$ were affected. Given the different findings of the prostate and endometrial studies, the relationship between FOXP1 and HIF expression remains to be clarified [15,51].

Increased nuclear FOXP1 expression correlated with expression of estrogen receptor-a in endometrial cancers [15]. In normal endometrial tissue, nuclear localisation of FOXP1 increased during the proliferative phase of the menstrual cycle, but only weak cytoplasmic staining was present during the secretory phase [15]. These observations suggest a potential interaction of FOXP1 with sex hormone receptors. However, increased FOXP1 expression is a predictor of relapse-free survival, independent of estrogen receptor expression status [15]. In this study of low risk, early stage endometrial cancers, there was no correlation with FOXP1 and overall survival [15].

2.1.4 Breast cancer-Nuclear FOXP1 protein expression was found to significantly correlate with estrogen receptor status and negatively correlate with epithelial growth factor receptors status in a study of 283 breast cancer patients [14]. The multivariate analysis showed that FOXP1 expression correlated with relapse free survival $(p=0.04)$ and a trend toward improved overall survival $(p=0.09)$ [14]. To investigate the possibility that FOXP1 may be regulated by estrogen, MCF-7 cells, an estrogen-dependent cell line, were treated with estrogen plus or minus tamoxifen [14]. No change was observed in FOXP1 expression in any of the experimental conditions [14]. The investigators concluded that FOXP1 might be a co-regulator of the estrogen receptor but did not depend on estrogen for its expression [14]. These results suggest that FOXP1 plays a critical role in the development of breast cancer, but the mechanism of its effect remains to be demonstrated. 
Collectively, there is significant correlative evidence indicating that FOXP1 may function as a tumour suppressor $[3,14,15]$. However, the direct evidence is limited. Although there are several interpretations to these results, the decreased apoptosis and increased cellular proliferation observed in $\mathrm{Foxpl}^{-/-}$cardiac tissues is consistent with a potential tumour suppressor role [10]. It is not known yet if it is the loss of FOXP1 that is important or if its loss is just a marker for loss of other genes. This will require evidence that functional loss of FOXP1 expression results in a predisposition to malignancy.

\subsection{FOXP1: oncogene}

Recent attention has focused on FOXP1 and its role in B-cell neoplasia. FOXP1 is expressed in cutaneous B-cell lymphomas, follicular cell lymphomas (FCL), marginal zone lymphomas (MZL), and diffuse large B-cell lymphoma (DLBCL). Its overexpression in subsets of DLBCL and MZL is associated with a poor patient prognosis [4,5,12,19,52]. A translocation of FOXP1 and the immunoglobulin heavy chain $(I G H)$ locus, $\mathrm{t}(3 ; 14)$, was initially described, resulting in increased expression of FOXP1. Subsequent studies have indicated that there are additional partners other than the $I G H$ locus involved in FOXPI translocations and in one case it was proposed that the breakpoint may reside within the coding region [13,53]. Increased FOXP1 expression correlates with trisomy 3 in some MZLs. The mechanism of FOXP1 overexpression remains to be elucidated in the majority of cases as not all strongly positive DLBCL display FOXP1 gene rearrangements or additional copies [54].

2.2.1 Diffuse large B-cell lymphoma-DLBCL comprises $\sim 40 \%$ of adult nonHodgkin lymphomas [4]. These are clinically, morphologically and genetically heterogeneous tumours composed of large B cells. Microarray studies have identified two main subgroups which can be distinguished by distinct gene-expression profiles as characteristic of either a germinal centre B-cell group (GCB-like), which has a 5-year survival of $60 \%$, or of an activated blood memory B-cell group (ABC-like or post-GC), which has a 5-year survival of 35\% [55-59]. A third subgroup, designated Type III DLBCL, has an intermediate prognosis and is likely heterogeneous in its composition [55].

A variety of B-cell tumours express abnormal levels of FOXP1, with gene profiling data suggesting this is especially in the case of ABC-DLBCL [4,13,60,61] (Figure 2). It is interesting that characteristic and recurrent genomic gains of chromosome 3 have also been reported to be associated with the ABC-like subtype of DLBCL [62]. In two separate studies, strong FOXP1 nuclear expression was correlated with a worse prognosis. In one study of DLBCL, in which FOXP1 staining of $>30 \%$ of the tumoural nuclei was considered FOXP1-positive, the median overall survival in the FOXP1-positive group was 1.6 years, as compared with 12.2 years in the FOXP1-negative group $(\mathrm{p}=0.0001)$ [4]. This study reported no association between FOXP1 expression and cytogenetic abnormalities [4]. A second study (in which FOXP1 nuclear positivity was categorised as negative, weak or strong) confirmed and extended this observation by correlating the effect of FOXP1 expression with overexpression of BCL-2 and MUM1/IRF4 [5]. They demonstrated that the group with strong FOXP1 nuclear staining had a median survival of 9.9 months, whereas patients with weak or negative staining had a significantly longer survival of up to 49 
months $(\mathrm{p}=0.015)$ [5]. In addition, when BCL-2 (a poor prognostic feature by itself ) and FOXP1 were co-expressed, the median survival of the BCL-2+FOXP1+ group was 8.0 months, whereas the BCL-2+FOXP1- group had a median OS of 28.4 months $(\mathrm{p}=0.021)$ [5]. High FOXP1 expression was not seen in tumours with the $\mathrm{t}(14 ; 18)$ BCL2-IgH translocation, a marker of the GC subgroup, suggesting the elevated expression is seen in the ABC or type III subgroups of DLBCL [5]. Both of these studies found FOXP1 to be an independent predictor for poor prognosis in a multivariant analysis. These results are in contrast to a study designed to validate the use of immunohistochemistry to define the GC and non-GC groups of DLBCL, which found no correlation between FOXP1 expression and prognosis [63]. In this negative study where $60 \%$ of patients were FOXP1+, a uniform cutoff of $30 \%$ nuclear staining was considered positive, but the intensity of FOXP1 staining was not noted [63]. In the studies that showed an association of FOXP1 with outcome, $18-$ $40 \%$ of the total cases were FOXP1+, depending on the system used $[4,5]$.

Despite the above discrepancy, data summarised in a recent review indicated that FOXP1 is an evolving and important entity in this more aggressive subtype [64]. FOXP1 has been classified among the 15 signature genes of ABC-DLBCL [102]. Of potential relevance in this context, NFAT family members are constitutively activated in B-cell tumours through bypass of the normal requirement for $\mathrm{Ca}^{2+}$-signal-induced nuclear translocation $[65,66]$. Recent reports have documented high and constitutive levels of NFAT2 in ABC-DBCL $[67,68]$. This suggests that FOXP1-NFAT cooperativity may be one possible pathway by which FOXP1 overexpression leads to a worse prognosis. The authors have recently investigated the expression of FOXP1 in ABC-like DLBCL cell lines by Western blotting and found that two smaller proteins, rather than the full-length protein, are atypically highly expressed compared to other B-cell lines (AH Banham, manuscript in preparation). As the JC12 monoclonal antibody recognises a common epitope, these FOXP1 isoforms are indistinguishable by immunohistochemistry. It is, therefore, possible that in ABC-like DLBCL the expression of these smaller proteins may interfere with or alter the normal function of the full-length protein. As DLBCL with strong FOXP1 expression have a worse prognosis than those that lack FOXP1 protein expression, it is also possible that these isoforms may have a function that is distinct from that of the full-length protein. These findings are supported by a study using mapping of common viral integration sites in avian nephroblastoma to identify potential human oncogenes, which identified foxPl as one of three most frequent targets [69]. None of the integration events affected foxP1 mRNA levels and all were clustered within the second coding exon, leading the authors to hypothesise that a virally truncated foxP1 protein might have a dominant-negative role and thus support malignant transformation. Further studies are required to determine the clinical significance of FOXP1 isoforms in DLBCL and other lymphomas. Also with the introduction of novel therapies, such as immunochemotherapy including the anti-CD20 monoclonal antibody rituximab, it will be interesting to see whether strong FOXP1 expression retains prognostic value. The only published study, to date, has reported that high levels of FOXP1 mRNA in meningeal lymphoma cells is associated with disease refractory to rituximab monotherapy [70]. 
2.2.2 Mucosal associated lymphoid tissue lymphoma-The mucosal associated lymphoid tissue (MALT) type of marginal zone B-cell lymphoma has been associated with increased FOXP1 expression. MALT lymphomas have a number of well-characterised translocations which target the NF- $\kappa \mathrm{B}$ pathway involving $M A L T 1$, a caspase-like protein; $A P I 2$, a member of the inhibitor of apoptosis gene family; and BCL10, a modulator of NF$\kappa \mathrm{B}$ signaling [71]. The $\mathrm{t}(3 ; 14)$, which places the FOXP1 gene under the control of the $I G H$ enhancer, was first described in a group MALT lymphomas that contained none of the "classic" MALT translocations [12]. In this series, 9 out of 91 MALT lymphomas had the $\mathrm{t}(3 ; 14)$ abnormality. It was demonstrated that MALT lymphomas with the $\mathrm{t}(3 ; 14)$, as well as some that had trisomy 3 , had elevated FOXPI transcription, as measured by quantitative reverse transcriptase polymerase chain reaction [12]. A follow-up study found that only 1 out of 11 cases of MALT lymphoma that were FOXP1-positive had $t(3 ; 14)$. However, these investigators also detected $t(3 ; 14)$ in only 1 out of 13 FOXP1-positive DLBCL, suggesting that, in an unselected sample, this was a rare translocation [13]. Although some cases with trisomy 3 had elevated levels of FOXP1, others did not, suggesting that gene amplification was insufficient to produce the elevated levels of FOXP1 observed [13]. In a series by the Mayo Clinic using 133 MALT lymphomas, no cases of the $\mathrm{t}(3 ; 14)$ were found [72]. Given that the previous reports of $\mathrm{t}(3 ; 14)$ in MALT lymphomas were from Europe or Japan, these authors suggested that regional variations in infectious agents implicated in the etiology of MALT lymphomas, such as Borellia burgdorferi or Chlamydophila psittaci, may explain their findings [72]. However, this seems unlikely as a study of 122 patients with extranodal marginal zone B-cell lymphomas of MALT subtype, from Germany, also found no cases carrying a breakpoint in the FOXP1 locus [53].

As with DLBCL, increased FOXP1 expression in MALT lymphoma is associated with poor prognosis. In a series of 70 cases where expression of FOXP1 was categorised as moderate or strong by nuclear staining, FOXP1-positivity was associated with a 5-year disease-free survival of 50\% in patients treated with curative intent as compared to 5-year disease-free survival of 100\% in the FOXP1-negative group ( $p=0.0004$ ) [60]. In addition, all five of the MALT lymphomas that transformed into DLBCL were among the eight patients with strong FOXP1 nuclear staining [60]. These data, in conjunction with the report of Haralambieva et al., in which 5 out of 33 extranodal DLBCL had genetic rearrangements involving FOXP1, suggest that dysregulation of FOXP1 in MALT lymphomas leads to transformation to a more aggressive DLBCL [53].

2.2.3 Other lymphomas-The data on FOXP1 expression in other lymphomas is limited, and its effect on their prognosis is unknown. FOXP1 is expressed in follicular cell lymphomas with 11 out of 13 cases having variable expression [52]. In the new WHOEORTC classification, cutaneous B-cell lymphomas are classified as primary cutaneous marginal zone B-cell lymphoma, primary cutaneous B-cell follicle center lymphoma (PCFCL) and primary cutaneous large B-cell lymphoma, leg type (PCLBCL-LT). In addition, lymphomas that do not fit in any of the above groups are called primary cutaneous large B-cell lymphoma, type other (PCLBCL-other). In a small series of cutaneous B-cell lymphomas, 4 out of 40 cases of PCFCL were FOXP1-positive and 3 out of 6 cases of PCLBCL-other were FOXP1-positive [19]. Intriguingly, in PCLBCT-LT, an entity 
associated with BCL-2 overexpression, 23 out of 29 case were FOXP1-positive [19]. In a larger series of PCFCL, 4 out of 104 cases were strongly positive and 25 out of 104 were weakly positive for FOXP1 [73]. Conversely, strong FOXP1 expression was seen in 26 out of 32 cases of PCLBCL-LT, and weak expression was seen in 5 out of 32 cases [73]. FOXP1 was correlated with shortened survival in PCFCL but there was no correlation with FOXP1 positivity and survival in PCLBCL-LT [73]. Unfortunately, neither report defined what percentage of cases co-expressed BCL-2 and FOXP1 [19,73].

No nuclear FOXP1 staining was observed in the Reed-Sternberg cells of 35 cases of classical Hodgkin's lymphoma [52]. Mediastinal B-cell lymphoma, a B-cell non-Hodgkin lymphoma that has a gene expression profile with characteristics common to classical Hodgkin's lymphoma, has also been reported to have decreased expression of FOXP1 [74]. Similar results were seen in lymphocyte-predominant Hodgkin's lymphoma, where 7 out of 8 cases were negative for FOXP1 staining [52]. FOXP1 expression was reported in the $\mathrm{CD} 20^{+} \mathrm{B}$-cell but rarely seen in the $\mathrm{CD} 30^{+} \mathrm{B}$-cell, while cells co-expressing CD30 and FOXP1 were noted within the T-cell areas of normal lymph nodes. These findings, along with the NFAT and FOXP3 results summarised above [34], suggest that investigating the role of FOXP1 in T-cells and in T-cell lymphomas may be of significant interest.

\section{Conclusion}

The data available at present is insufficient to conclusively determine whether FOXP1 functions as a tumour suppressor or as an oncogene, depending on the cell type, on its interacting partners or its subcellular localisation. Although the published data support a role for FOXP1 as an oncogene in lymphoma, it may be that only smaller isoforms exhibit this role and that they do so by abrogating the tumour suppressor function of the full-length protein. Both proposed functions for FOXP1 in tumourigenesis require more experimental proof before either role can be claimed with certainty. It is clear that FOXP1 overexpression defines a group of poor-prognosis lymphomas, but the mechanism and molecular consequences of this effect remain to be elucidated. Given the current level of understanding, the first clinical applications of FOXP1 expression will likely be in the setting of patient selection and stratification in clinical studies. But as knowledge increases, FOXP1-directed therapies have significant potential.

\section{Expert opinion}

The overexpression, mislocalisation or loss of FOXP1 in a wide variety of cancers suggests that FOXP1 may be a therapeutic target or may be used to guide therapy. The most significant barriers to using FOXP1 are, firstly, that most of the data implicating FOXP1 in malignancy is essentially based on altered expression patterns and correlation with clinical outcome. Secondly, there is little information available on its normal biological role to enable a functional comparison of FOXP1 proteins in malignant cells. Thirdly, it is unclear whether FOXP1 can act as either, or both, a tumour suppressor and oncogene, and whether or not FOXP1 isoforms play a key role in lymphomas. Further studies are clearly needed before therapeutic targeting is likely to become a reality. 
If $F O X P 1$ proves to be a tumour suppressor, gene therapy to replace $F O X P 1$ is a possibility. As Foxpl heterozygous mice are normal in every aspect in which they have been examined, then it is possible that demethylating agents may be able to restore FOXPl expression, if methylation is a key mechanism underlying silencing of the second allele. Given that overexpression of FOXP1 is potentially a transforming event, any FOXP1-based gene therapy would require a delivery system that would only target affected cells. Additionally, gene therapy has the risk of insertion into an endogenous locus, resulting in transformation [75]. Although targeted viral delivery systems are under development, FOXP1-based gene therapy to restore expression levels is unlikely to be of clinical use in the next five years [76].

The function of some transcription factors, including NFAT, FOXO and the signal transducer and activator of transcription (STATs), are regulated by nuclear-cytoplasmic localisation $[77,78]$. NFAT family members must be dephosphorylated by the calcineurin phosphatase complex as a requirement for $\mathrm{Ca}^{2+}$ signal-induced nuclear translocation [55]. In the case of the FOXO transcription factors, activation of the phosphatidylinositol 3-kinase (PI3K)/AKT pathway leads to cytoplasmic localisation and inactivation of FOXO-dependent transcription [78]. Conversely, growth factor-mediated tyrosine and serine phosphorylation of STATs is required for their nuclear entry and subsequent transactivation [77]. Although nucleocytoplasmic shuttling of FOXP1 has not been documented in normal cells, its aberrant localisation has been observed within the cytoplasm of malignant cells [3]. This event is much more frequent in epithelial malignancies, although rare cytoplasmic FOXP1 expression has been described in both Hodgkin's lymphomas and DLBCL [5,52]. If cytoplasmic localisation of FOXP1 proves to be of functional significance, targeting the upstream signal transduction pathways or alternative mechanisms that regulate its localisation may be a therapeutic strategy to restore nuclear expression.

The loss of FOXPI alone, or in combination with the other candidate tumour suppressors on $3 p$, may also be used as a marker to guide targeted therapy. One potential approach to this problem is to apply a small interfering RNAi screen to cells that are well characterised for loss of FOXP1 and the other 3p tumours suppressors. In this type of screen, genes whose "knockdown" inhibit growth and are "druggable" could then be validated with targeted therapies such as small-molecule inhibitors and antibodies. These types of approaches would to lead to specific targets that could quickly be translated into therapies in cancers that have loss of FOXP1.

Similar strategies may be employed to target FOXPl if it functions as an oncogene. RNAi screens to determine targets that lead to cytoplasmic localisation of FOXP1 may identify a specific regulatory molecule (e.g., a kinase) that could be inhibited. In the case of lymphomas that overexpress FOXP1, functional inactivation by cytoplasmic localisation may lead to growth arrest or to rendering the cells more susceptible to other therapies.

However, it is also possible that mislocalisation of FOXP1 might have adverse consequences, for example through the mislocalisation of its binding partners. The smaller FOXP1 isoforms in ABC-like DLBCL cell lines may represent a more promising target for therapy if they are found to contribute to the disease process. A complementary strategy would be to identify pathways that either co-operate with FOXP1 in transformation or that 
include FOXP1, such as those facilitated by NFAT, NF- $\kappa$ B or BCL-2 in high-risk groups of lymphomas. These data could be used in multiple ways. If the interactions between FOXP1 and other transcription factors are direct, screens for small molecules that interrupt the protein-protein interactions, such as that between FOXP1 and NFAT2, may produce therapeutic candidates. This same type of approach could be used to identify inhibitors that block the binding of FOXP1 and DNA or the dimerisation of FOXP family members, which is required for their DNA binding. However, FOXP1-targeted approaches may have offtarget effects in vivo as well, given the structural similarities and broad expression patterns among the FOXP subfamily members.

Most of the potential impact of FOXP1 therapeutic development requires significant advances in our understanding of the biology of FOXP1. However, the poor prognosis of $d e$ novo DLBCL patients co-expressing FOXP1 and BCL-2 suggests at least one approach using therapies already under investigation. In one study, BCL-2+FOXP1-negative and BCL-2+FOXP1-positive DLBCL had a significant difference in median survival of 28.4 months versus 8.0 months, respectively [5]. BCL-2 overexpression is known to be a marker of poor prognosis in a number of cancers, and BCL-2 antisense oligonucleotide (oblimersen sodium) is a strategy under clinical investigation [79]. BCL-2 antisense has been shown to improve outcomes when used in combination with dacarbazine in melanoma. As BCL-2 overexpressing de novo DLBCL that co-overexpress FOXP1 have a worse prognosis, one could envision a trial of chemotherapy +/- BCL-2 antisense that was stratified for FOXP1 expression. This type of study would not only provide efficacy data for BCL-2 antisense in lymphoma, but may also give insight into whether or not FOXP1 expression is important for patient selection of BCL-2 targeted therapy. Additionally, it would potentially provide in vivo data on the potentially additive risk of FOXP1 and BCL-2 co-expression its significance in de novo DLBCL.

\section{Bibliography}

Papers of special note have been highlighted as either of interest $(\bullet)$ or of considerable interest $(\bullet \bullet)$ to readers.

1. HECHT JL, ASTER JC. Molecular biology of Burkitt's lymphoma. J. Clin. Oncol. 2000; 18(21): 3707-3721. [PubMed: 11054444]

2. SCHUSTER MB, PORSE BT. C/EBPa: a tumour suppressor in multiple tissues? Biochim. Biophys. Acta. 2006; 1766(1):88-103. [PubMed: 16616425]

3. BANHAM AH, BEASLEY N, CAMPO E, et al. The FOXP1 winged helix transcription factor is a novel candidate tumor suppressor gene on chromosome 3p. Cancer Res. 2001; 61(24):8820-8829. -• This study was the first to report the abnormal expression patterns of Foxp1 in solid tumors and also its overexpression in a subset of diffuse large B-cell lymphomas. [PubMed: 11751404]

4. BANHAM AH, CONNORS JM, BROWN PJ, et al. Expression of the FOXP1 transcription factor is strongly associated with inferior survival in patients with diffuse large B-cell lymphoma. Clin. Cancer Res. 2005; 11(3):1065-1072. • Using the JC12 monoclonal antibody, FOXP1 protein expression was analyzed by immunohistochemistry using a DLBCL tissue microarray constructed from 101 previously untreated de novo cases obtained at a single institution. Multivariate analysis confirmed that FOXP1 expression has prognostic significance in patients with de novo DLBCL, strongly suggesting that FOXP1 may be important in DLBCL pathogenesis. [PubMed: 15709173]

5. BARRANS SL, FENTON JA, BANHAM A, OWEN RG, JACK AS. Strong expression of FOXP1 identifies a distinct subset of diffuse large B-cell lymphoma (DLBCL) patients with poor outcome. Blood. 2004; 104(9):2933-2935. [PubMed: 15238418] 
6. CARLSSON P, MAHLAPUU M. Forkhead transcription factors: key players in development and metabolism. Dev. Biol. 2002; 250(1):1-23. [PubMed: 12297093]

7. KATOH M, KATOH M. Human FOX gene family (Review). Int. J. Oncol. 2004; 25(5):1495-1500. [PubMed: 15492844]

8. HU H, WANG B, BORDE M, et al. Foxp1 is an essential transcriptional regulator of B cell development. Nat. Immunol. 2006; 7(8):819-826. •• This outstanding paper clearly identifies Foxp1 as a key regulator among the network of transcription factors that regulate B-cell development. The authors present convincing evidence that Foxp1 controls the B-cell specific expression of the Rag1 and Rag2 genes responsible for VDJ gene recombination. [PubMed: 16819554]

9. SHU W, YANG H, ZHANG L, LU MM, MORRISEY EE. Characterization of a new subfamily of winged-helix/forkhead (Fox) genes that are expressed in the lung and act as transcriptional repressors. J. Biol. Chem. 2001; 276(29):27488-27497. [PubMed: 11358962]

10. WANG B, WEIDENFELD J, LU MM, et al. Foxp1 regulates cardiac outflow tract, endocardial cushion morphogenesis and myocyte proliferation and maturation. Development. 2004; 131(18): 4477-4487. [PubMed: 15342473]

11. SHI C, ZHANG X, CHEN Z, et al. Integrin engagement regulates monocyte differentiation through the forkhead transcription factor Foxp1. J. Clin. Invest. 2004; 114(3):408-418. [PubMed: 15286807]

12. STREUBEL B, VINATZER U, LAMPRECHT A, RADERER M, CHOTT A. T(3;14)(p14.1;q32) involving IGH and FOXP1 is a novel recurrent chromosomal aberration in MALT lymphoma. Leukemia. 2005; 19(4):652-658. [PubMed: 15703784]

13. WLODARSKA I, VEYT E, DE PAEPE P, et al. FOXP1, a gene highly expressed in a subset of diffuse large B-cell lymphoma, is recurrently targeted by genomic aberrations. Leukemia. 2005; 19(8):1299-1305. [PubMed: 15944719]

14. FOX SB, BROWN P, HAN C, et al. Expression of the forkhead transcription factor FOXP1 is associated with estrogen receptor $\mathrm{a}$ and improved survival in primary human breast carcinomas. Clin. Cancer Res. 2004; 10(10):3521-3527. [PubMed: 15161711]

15. GIATROMANOLAKI A, KOUKOURAKIS MI, SIVRIDIS E, et al. Loss of expression and nuclear/cytoplasmic localization of the FOXP1 forkhead transcription factor are common events in early endometrial cancer: relationship with estrogen receptors and HIF-1a expression. Mod. Pathol. 2006; 19(1):9-16. [PubMed: 16258506]

16. LI C, TUCKER PW. DNA-binding properties and secondary structural model of the hepatocyte nuclear factor 3/fork head domain. Proc. Natl. Acad. Sci. USA. 1993; 90(24):11583-11587. [PubMed: 8265594]

17. HUEBNER K. Tumor suppressors on 3p: a neoclassic quartet. Proc. Natl. Acad. Sci. USA. 2001; 98(26):14763-14765. [PubMed: 11752421]

18. ZABAROVSKY ER, LERMAN MI, MINNA JD. Tumor suppressor genes on chromosome 3p involved in the pathogenesis of lung and other cancers. Oncogene. 2002; 21(45):6915-6935. [PubMed: 12362274]

19. HOEFNAGEL JJ, MULDER MM, DREEF E, et al. Expression of B-cell transcription factors in primary cutaneous B-cell lymphoma. Mod. Pathol. 2006; 19(9):1270-1276. [PubMed: 16778825]

20. CRESPI BJ. Sly FOXP2: genomic conflict in the evolution of language. Trends Ecol. Evol. 2007

21. LAI CS, FISHER SE, HURST JA, VARGHA-KHADEM F, MONACO AP. A forkhead-domain gene is mutated in a severe speech and language disorder. Nature. 2001; 413(6855):519-523. [PubMed: 11586359]

22. CHATILA TA, BLAESER F, HO N, et al. JM2, encoding a fork head-related protein, is mutated in X-linked autoimmunity-allergic disregulation syndrome. J. Clin. Invest. 2000; 106(12):R75-R81. [PubMed: 11120765]

23. BENNETT CL, OCHS HD. IPEX is a unique X-linked syndrome characterized by immune dysfunction, polyendocrinopathy, enteropathy, and a variety of autoimmune phenomena. Curr. Opin. Pediatr. 2001; 13(6):533-538. [PubMed: 11753102]

24. ZIEGLER SF. FOXP3: of mice and men. Annu. Rev. Immunol. 2006; 24:209-226. [PubMed: $16551248]$ 
25. TEUFEL A, WONG EA, MUKHOPADHYAY M, MALIK N, WESTPHAL H. FoxP4, a novel forkhead transcription factor. Biochim. Biophys. Acta. 2003; 1627(2-3):147-152. [PubMed: 12818433]

26. LI S, ZHOU D, LU MM, MORRISEY EE. Advanced cardiac morphogenesis does not require heart tube fusion. Science. 2004; 305(5690):1619-1622. [PubMed: 15361625]

27. DE LA POMPA JL, TIMMERMAN LA, TAKIMOTO H, et al. Role of the NF-ATc transcription factor in morphogenesis of cardiac valves and septum. Nature. 1998; 392(6672):182-186. [PubMed: 9515963]

28. WANG B, LIN D, LI C, TUCKER P. Multiple domains define the expression and regulatory properties of Foxp1 forkhead transcriptional repressors. J. Biol. Chem. 2003; 278(27):2425924268. [PubMed: 12692134]

29. LI S, WEIDENFELD J, MORRISEY EE. Transcriptional and DNA binding activity of the Foxp1/2/4 family is modulated by heterotypic and homotypic protein interactions. Mol. Cell Biol. 2004; 24(2):809-822. [PubMed: 14701752]

30. COFFER PJ, BURGERING BM. Forkhead-box transcription factors and their role in the immune system. Nat. Rev. Immunol. 2004; 4(11):889-899. [PubMed: 15516968]

31. SCHUBERT LA, JEFFERY E, ZHANG Y, RAMSDELL F, ZIEGLER SF. Scurfin (FOXP3) acts as a repressor of transcription and regulates T cell activation. J. Biol. Chem. 2001; 276(40):3767237679. [PubMed: 11483607]

32. BETTELLI E, DASTRANGE M, OUKKA M. Foxp3 interacts with nuclear factor of activated T cells and NF- $\kappa$ B to repress cytokine gene expression and effector functions of T helper cells. Proc. Natl. Acad. Sci. USA. 2005; 102(14):5138-5143. [PubMed: 15790681]

33. FOX AH, LAM YW, LEUNG AK, et al. Paraspeckles: a novel nuclear domain. Curr. Biol. 2002; 12(1):13-25. [PubMed: 11790299]

34. WU Y, BORDE M, HEISSMEYER V, et al. FOXP3 controls regulatory T cell function through cooperation with NFAT. Cell. 2006; 126(2):375-387. •• In an elegant series of structural and in vivo experiments, the authors show that Foxp family proteins interact with NFAT2 to bind to "composite" sites within the IL-2 promoter to repress its activity. [PubMed: 16873067]

35. RAO A, LUO C, HOGAN PG. Transcription factors of the NFAT family: regulation and function. Annu. Rev. Immunol. 1997; 15:707-747. [PubMed: 9143705]

36. CRABTREE GR, OLSON EN. NFAT signaling: choreographing the social lives of cells. Cell. 2002; 109(Suppl.):S67-S79. [PubMed: 11983154]

37. HANS CP, WEISENBURGER DD, GREINER TC, et al. Expression of PKC- $\beta$ or cyclin D2 predicts for inferior survival in diffuse large B-cell lymphoma. Mod. Pathol. 2005; 18(10):13771384. [PubMed: 15920548]

38. BUSSLINGER M. Transcriptional control of early B cell development. Annu. Rev. Immunol. 2004; 22:55-79. [PubMed: 15032574]

39. MURRE C. Helix-loop-helix proteins and lymphocyte development. Nat. Immunol. 2005; 6(11): 1079-1086. [PubMed: 16239924]

40. SAKAGUCHI S, SAKAGUCHI N, SHIMIZU J, et al. Immunologic tolerance maintained by $\mathrm{CD} 25^{+} \mathrm{CD} 4^{+}$regulatory $\mathrm{T}$ cells: their common role in controlling autoimmunity, tumor immunity, and transplantation tolerance. Immunol. Rev. 2001; 182:18-32. [PubMed: 11722621]

41. SHEVACH EM. CD $4{ }^{+} \mathrm{CD} 25^{+}$suppressor T cells: more questions than answers. Nat. Rev. Immunol. 2002; 2(6):389-400. [PubMed: 12093005]

42. FONTENOT JD, GAVIN MA, RUDENSKY AY. Foxp3 programs the development and function of $\mathrm{CD} 4^{+} \mathrm{CD} 25^{+}$regulatory T cells. Nat. Immunol. 2003; 4(4):330-336. [PubMed: 12612578]

43. HORI S, NOMURA T, SAKAGUCHI S. Control of regulatory T cell development by the transcription factor Foxp3. Science. 2003; 299(5609):1057-1061. [PubMed: 12522256]

44. KHATTRI R, COX T, YASAYKO SA, RAMSDELL F. An essential role for Scurfin in $\mathrm{CD}^{+}{ }^{+} \mathrm{CD} 25^{+} \mathrm{T}$ regulatory cells. Nat. Immunol. 2003; 4(4):337-342. [PubMed: 12612581]

45. BANHAM AH, POWRIE FM, SURI-PAYER E. FOXP3 ${ }^{+}$regulatory T cells: Current controversies and future perspectives. Eur. J. Immunol. 2006; 36(11):2832-2836. [PubMed: 17051620] 
46. MARSON A, KRETSCHMER K, FRAMPTON GM, et al. Foxp3 occupancy and regulation of key target genes during T-cell stimulation. Nature. 2007; 445(7130):931-935. [PubMed: 17237765]

47. SHU W, LU MM, ZHANG Y, et al. Foxp2 and Foxp1 cooperatively regulate lung and esophagus development. Development. 2007; 134(10):1991-2000. [PubMed: 17428829]

48. DUMON KR, ISHII H, FONG LY, et al. FHIT gene therapy prevents tumor development in Fhitdeficient mice. Proc. Natl. Acad. Sci. USA. 2001; 98(6):3346-3351. [PubMed: 11248081]

49. XIAN J, AITCHISON A, BOBROW L, et al. Targeted disruption of the $3 p 12$ gene, Dutt1/Robo1, predisposes mice to lung adenocarcinomas and lymphomas with methylation of the gene promoter. Cancer Res. 2004; 64(18):6432-6437. [PubMed: 15374951]

50. SHIMIZU K, KATO A, HINOTSUME D, et al. Reduced expressions of Foxp1 and Rassf1a genes in lung adenocarcinomas induced by $\mathrm{N}$-nitrosobis(2-hydroxypropyl)-amine in rats. Cancer Lett. 2006; 236(2):186-190. [PubMed: 16023287]

51. BANHAM AH, BODDY J, LAUNCHBURY R, et al. Expression of the forkhead transcription factor FOXP1 is associated both with hypoxia inducible factors (HIFs) and the androgen receptor in prostate cancer but is not directly regulated by Androgens or hypoxia. Prostate. 2007

52. BROWN P, MARAFIOTI T, KUSEC R, BANHAM AH. The FOXP1 transcription factor is expressed in the majority of follicular lymphomas but is rarely expressed in classical and lymphocyte predominant Hodgkin's lymphoma. J. Mol. Histol. 2005; 36(4):249-256. [PubMed: 16200457]

53. HARALAMBIEVA E, ADAM P, VENTURA R, et al. Genetic rearrangement of FOXP1 is predominantly detected in a subset of diffuse large B-cell lymphomas with extranodal presentation. Leukemia. 2006; 20(7):1300-1303. [PubMed: 16673020]

54. BARRANS SL, FENTON JAL, VENTURA R, et al. Deregulated over expression of FOXP1 protein in diffuse large B-cell lymphoma does not occur as a result of gene rearrangement. Haematologica. 2007 In Press.

55. The International non-Hodgkin's lymphoma prognostic factors project. N. Engl. J. Med. 1993; 329(14):987-994. A predictive model for aggressive non-Hodgkin's lymphoma. [PubMed: 8141877]

56. ALIZADEH AA, EISEN MB, DAVIS RE, et al. Distinct types of diffuse large B-cell lymphoma identified by gene expression profiling. Nature. 2000; 403(6769):503-511. [PubMed: 10676951]

57. ATKINS CD. A predictive model for non-Hodgkin's lymphoma. N. Engl. J. Med. 1994; 330(8): 574-575. [PubMed: 8302331]

58. ROSENWALD A, WRIGHT G, CHAN WC, et al. The use of molecular profiling to predict survival after chemotherapy for diffuse large-B-cell lymphoma. N. Engl. J. Med. 2002; 346(25): 1937-1947. [PubMed: 12075054]

59. SHIPP MA, ROSS KN, TAMAYO P, et al. Diffuse large B-cell lymphoma outcome prediction by gene-expression profiling and supervised machine learning. Nat. Med. 2002; 8(1):68-74. [PubMed: 11786909]

60. SAGAERT X, DE PAEPE P, LIBBRECHT L, et al. Forkhead box protein P1 expression in mucosa-associated lymphoid tissue lymphomas predicts poor prognosis and transformation to diffuse large B-cell lymphoma. J. Clin. Oncol. 2006; 24(16):2490-2497. [PubMed: 16636337]

61. SHAFFER AL, ROSENWALD A, STAUDT LM. Lymphoid malignancies: the dark side of B-cell differentiation. Nat. Rev. Immunol. 2002; 2(12):920-932. • Gene expression profiling was used to document that FOXP1 mRNA is upregulated in response to normal B-cell activation and is highly expressed in the poor-prognosis activated subtype of DLBCL. [PubMed: 12461565]

62. BEA S, ZETTL A, WRIGHT G, et al. Diffuse large B-cell lymphoma subgroups have distinct genetic profiles that influence tumor biology and improve gene-expression-based survival prediction. Blood. 2005; 106(9):3183-3190. [PubMed: 16046532]

63. HANS CP, WEISENBURGER DD, GREINER TC, et al. Confirmation of the molecular classification of diffuse large B-cell lymphoma by immunohistochemistry using a tissue microarray. Blood. 2004; 103(1):275-282. [PubMed: 14504078]

64. DE PAEPE P, DE WOLF-PEETERS C. Diffuse large B-cell lymphoma: a heterogeneous group of non-Hodgkin lymphomas comprising several distinct clinicopathological entities. Leukemia. 2007; 21(1):37-43. [PubMed: 17039226] 
65. GILMORE TD, KALAITZIDIS D, LIANG MC, STARCZYNOWSKI DT. The c-Rel transcription factor and B-cell proliferation: a deal with the devil. Oncogene. 2004; 23(13):2275-2286. [PubMed: 14755244]

66. MARAFIOTI T, POZZOBON M, HANSMANN ML, et al. The NFATc1 transcription factor is widely expressed in white cells and translocates from the cytoplasm to the nucleus in a subset of human lymphomas. Br. J. Haematol. 2005; 128(3):333-342. [PubMed: 15667535]

67. FU L, LIN-LEE YC, PHAM LV, et al. Constitutive NF- $\kappa B$ and NFAT activation leads to stimulation of the BLyS survival pathway in aggressive B-cell lymphomas. Blood. 2006; 107(11): 4540-4548. [PubMed: 16497967]

68. PHAM LV, TAMAYO AT, YOSHIMURA LC, LIN-LEE YC, FORD RJ. Constitutive NF- $\kappa$ B and NFAT activation in aggressive B-cell lymphomas synergistically activates the CD154 gene and maintains lymphoma cell survival. Blood. 2005; 106(12):3940-3947. [PubMed: 16099873]

69. PAJER P, PECENKA V, KRALOVA J, et al. Identification of potential human oncogenes by mapping the common viral integration sites in avian nephroblastoma. Cancer Res. 2006; 66(1):7886. [PubMed: 16397219]

70. RUBENSTEIN JL, FRIDLYAND J, ABREY L, et al. Phase I study of intraventricular administration of rituximab in patients with recurrent CNS and intraocular lymphoma. J. Clin. Oncol. 2007

71. FARINHA P, GASCOYNE RD. Molecular pathogenesis of mucosa-associated lymphoid tissue lymphoma. J. Clin. Oncol. 2005; 23(26):6370-6378. [PubMed: 16155022]

72. REMSTEIN ED, DOGAN A, EINERSON RR, et al. The incidence and anatomic site specificity of chromosomal translocations in primary extranodal marginal zone B-cell lymphoma of mucosaassociated lymphoid tissue (MALT lymphoma) in North America. Am. J. Surg. Pathol. 2006; 30(12):1546-1553. [PubMed: 17122510]

73. SENFF NJ, HOEFNAGEL JJ, JANSEN PM, et al. Reclassification of 300 primary cutaneous Bcell lymphomas according to the new WHO-EORTC classification for cutaneous lymphomas: comparison with previous classifications and identification of prognostic markers. J. Clin. Oncol. 2007

74. SAVAGE KJ, MONTI S, KUTOK JL, et al. The molecular signature of mediastinal large B-cell lymphoma differs from that of other diffuse large B-cell lymphomas and shares features with classical Hodgkin lymphoma. Blood. 2003; 102(12):3871-3879. [PubMed: 12933571]

75. HACEIN-BEY-ABINA S, VON KALLE C, SCHMIDT M, et al. LMO2-associated clonal T cell proliferation in two patients after gene therapy for SCID-X1. Science. 2003; 302(5644):415-419. [PubMed: 14564000]

76. YANG L, BAILEY L, BALTIMORE D, WANG P. Targeting lentiviral vectors to specific cell types in vivo. Proc. Natl. Acad. Sci. USA. 2006; 103(31):11479-11484. [PubMed: 16864770]

77. BROMBERG J, DARNELL JE JR. The role of STATs in transcriptional control and their impact on cellular function. Oncogene. 2000; 19(21):2468-2473. [PubMed: 10851045]

78. STITT TN, DRUJAN D, CLARKE BA, et al. The IGF-1/PI3K/Akt pathway prevents expression of muscle atrophy-induced ubiquitin ligases by inhibiting FOXO transcription factors. Mol. Cell. 2004; 14(3):395-403. [PubMed: 15125842]

79. BEDIKIAN AY, MILLWARD M, PEHAMBERGER H, et al. Bcl-2 antisense (oblimersen sodium) plus dacarbazine in patients with advanced melanoma: the Oblimersen Melanoma Study Group. J. Clin. Oncol. 2006; 24(29):4738-4745. [PubMed: 16966688]

\section{Websites}

101. http://symatlas.gnf.org

102. http://lymphochip.nih.gov/signaturedb/query.htm

Expert Opin Ther Targets. Author manuscript; available in PMC 2015 January 02. 
Key issues to address in future include:

- How FOXP1 subcellular localisation is regulated and if the changes in localisation are physiologically relevant as would be predicted.

- What transcription factors or cofactors other than NFAT and CtBP1 interact with FOXP1 and how are these interactions regulated.

- What are the transcriptional targets of FOXP1?

- Experimental data is needed to determine if FOXP1 functions as an oncogene or a tumour suppressor or both and to investigate the role of FOXP1 isoforms.

- Are there other cancers where FOXP1 expression has prognostic implications?

- Can FOXP1 expression status be used as a guide to therapy? 


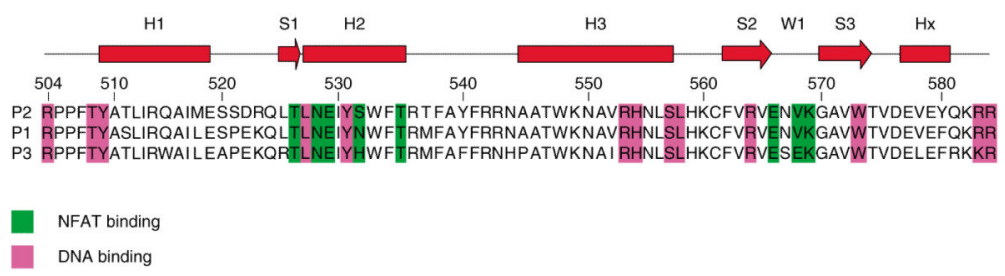

Figure 1. FOXP forkhead domains and NFAT-contacting residues

Upper, schematic secondary structure of the FOXP1 forkhead domain. H1, H2, H3 denote alpha-helix regions; S1, S2, S3 represent beta-strands; W1: the wing domain Lower, alignment of FOXP1-3 with NFAT-binding and DNA-binding residues indicated in green and violet, respectively.

Adapted and reprinted from [34] by permission from Elsevier Publishing, Ltd, London. NFAT: Nuclear factor of activated T cells. 


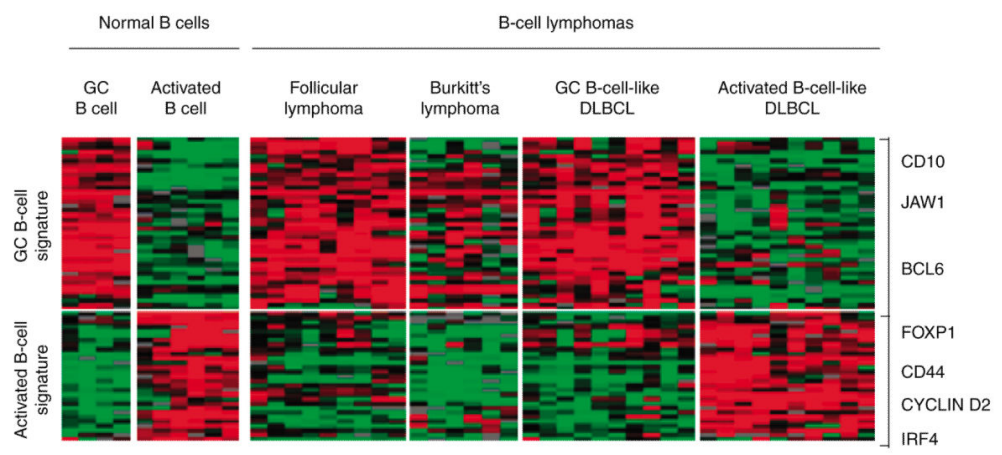

Figure 2. FOXP1 expression in ABC-DLBCL

$F O X P 1$ overexpression is a diagnostic genetic marker that has been used to categorise the activated B-cell-like subtype of diffuse large B-cell lymphoma (ABC-DLBCL). Microarray technology was used to determine gene-expression profiles of normal B cells as well as several types of mature B-cell lymphomas. The data is depicted as a "heat map" wherein the color red represents a high relative level of gene expression (RNA transcription), the color green represents a low relative level of gene expression, and black represents an average baseline expression. Each column denotes an individual RNA sample derived from either a normal B-cell patient sample or a malignant B-cell tumour. Each row denotes the expression of a single gene. A 16-fold range of gene expression is shown. Indicated is a subset of genes differentially expressed in GC B cells of secondary lymphoid follicles when compared to mitogenically activated B-cells derived from peripheral blood. The genes CD10, JAW1, and $B C L 6$, for example, seem to define an essential GC B-cell gene-expression signature, which is shared by some lymphomas - namely, follicular lymphoma, Burkitt's lymphoma, and the GC B-cell-like subset of diffuse large B-cell lymphoma (GC-DLBCL). In contrast, the genes CD44, Cyclin D2, IRF4, and FOXP1 - which are expressed at a higher relative level in mitogenically activated peripheral-blood B cells than in GC B cells - uniquely identify the ABC-like DLBCL subgroup.

Adapted from [61] with permission from Macmillan Publishers Ltd.

DLBLC: Diffuse large B-cell lymphoma; GC: Germinal centre. 